\title{
STATUS AND NEEDS OF ASTROMETRY
}

James A. Hughes

U.S. Naval Observatory, Washington, D.C. 20390

\section{ABSTRACT}

A review will be presented of the status of the fundamental reference system. Mention will be made of the problems limiting the accuracy of transit circles and photographic astrometry and attempts at possible improvements in the near future. The progress in the preparation of the FK5 will be briefly mentioned, as will be the various methods used to approximate an inertial reference frame. Needs for higher accuracy in Astronomy fall in the area of better modeling of the Earth's motion (rotation, nutation, precession, the ecliptic, etc.) as well as a parallax and proper motion determination for galactic structure studies, stellar dynamics and stellar evolution. It will be assumed that interferometric binary star studies will be discussed elsewhere.

\section{INTRODUCTION}

Although astrometry may be quite simply defined as, "the measurement of celestial positions and motions", this definition, as is commonly the case with definitions, does not really tell one anything about what is actually measured or how the measurement is accomplished. In actual fact the road leading to a celestial "position" or "motion" is tortuous indeed, involving, as it does, the search for some idealized "inertial reference system".

From an operational point of view it is convenient to divide astrometry into two conceptually trivial parts, namely, (1) measuring large angles, and (2) measuring small angles. The implications of this somewhat embarrassing division, however, are not trivial with regard to the end which is sought, its precision and accuracy, the techniques and instrumentation employed, and perhaps the universal significance of the result. 
In general, small angle measurements are concerned with differential positions or motions whereby any convenient point or combination of points is used to explicitly or implicitly define an origin. Examples of these kinds of measurements include:

1. Differential transit circle zone star catalogs

2. Photographic star catalogs

3. Parallax determinations

4. Binary Star observations

5. Cluster Studies

6. Proper Motion Surveys

7. Monitoring Earth rotation parameters.

Evidently the bulk of astrometric work is included in this category.

There are few examples of classical, large angle, optical measurements. Two which come to mind are the astrolabe and transit (or meridian) circle. Since,for all practical purposes, the current approximations to an inertial system rest upon the latter, only fundamental catalogs produced by transit circles w11l be considered here. The word "fundamental" is used in the sense that the observing program leading to such a catalog independently determines its own origins, and positions with respect to them, by measuring large arcs in an absolute instrumental system.

These origins have been historically the equinox and equator ${ }^{1}$. The reason for the choice of non-observable, dynamic zero points is simply that astrometry has been actually concerned with several problems simultaneously, i.e., the determination of, (1) the positions and intrinsic motions of celestial objects, (2) the "induced" motions from, e.g., parallax and aberration, (3) the "dynamical" motion of the observing platform, Earth, and (4) the "geophysical" motion of the mantle of the earth. Given these considerations the choice of origins was natural and useful. The use of a dynamic origin, however, carries with it the concomitant responsibility for keeping careful records of the theories and numerical constants used in any program. With this precaution a dynamic origin is, in principle and in practice, as good as any other. It should be emphasized that, observable or not, the equinox and 
equator are obtained through observation. When a star is assigned a right ascension, $x$, in a fundamental catalog, the statement being made is not so much that the star is $x$ hours from the equinox, but is rather its somewhat Orwellian Inverse, that the equinox of that catalog is $-x$ hours from that particular star.

The equinox and the equator are determined in practice by observations of solar system objects. A particularly appealing method involves observations of asterolds which appear to be star-like and which are observed optically at night. Although the method has had its difficulties it appears that much of the problem lies in selection effects, i.e. which minor planets are observed and when they are observed. Theoretical calculations ${ }^{2}$ have shown that with careful planning of the program, and using realistic magnitude 1imits, at a reasonably clear site, the errors are approximately \pm "04 and $\pm ! 004$ in the equinox and equator respectively, where the mean error of one observation is taken as \pm 0 ":25. Equivalent numbers are, for the Sun, \pm "06 and $\pm ! \cdot 02$, and for the planets Mercury, Venus and Mars, about \pm "15 and \pm "06.

\section{THE STATUS OF THE FUNDAMENTAL SYSTEM}

The principal fundamental coordinate system currently in use over the entire sky is defined by the positions and motions of 1,535 stars given in the Fourth Fundamental Catalog, $\mathrm{FK}_{4}{ }^{3}$. It is a compilation of results from over two hundred observational catalogs covering a time span of some 100 years. Its mean epochs are; in $\alpha, 1935$, and in $\delta, 1925$. Of the contributing catalogs some $40 \%$ are fundamental. The remaining catalogs are differential, but very useful for internal adjustements during the compilation. No photographic catalogs were used.

The production of a fundamental coordinate system such as the FK4 is an intricate process involving many concepts and statistical procedures as well as the judgement of the compiler. For example, a particularly vexing problem involves the inclusion of older, less precise catalogs to increase the weight of the proper motions. Where is the optimum cutoff? This problem is being overcome as rapidly as instrumental improvements and the scale of stellar motions allow. 
The formal errors of the FK4 are given in terms of two, presumably independent components. The first, derived for the individual stars, is measured by the dispersion of the information for particular stars among all contributing catalogs. The second, termed the "system error", is derived by consideration of the systematic differences among the contributing fundamental (i.e. independent) catalogs. System errors apply to all stars. The total squared error is then the sum of the squares of the two components. Errors are given for the positions at mean epoch and for the proper motions.

Regarding the magnitude of these errors; if one were to compute the position of some "average" FK4 star today, then according to Lederle ${ }^{4}$ the error would be, in seconds of arc,

\begin{tabular}{|c|c|c|c|c|c|c|}
\hline \multicolumn{3}{|c|}{ Northern Sky } & \multicolumn{3}{|c|}{ Southern Sky } \\
\hline & Ind. & Sys. & Tot. & Ind. & Sys. & Tot. \\
\hline$\alpha$ & .07 & .06 & .09 & .15 & .12 & .19 \\
\hline$\delta$ & .08 & .04 & .09 & .14 & .07 & .16 \\
\hline
\end{tabular}

This represents the errors at mean epochs $(\alpha, 1935, \delta, 1925)$, plus the accumulated error due to uncertainty in the proper motions since the mean epochs.

Frankly, one hesitates to present such tables, but such a presentation is probably expected here. The difficulty is that there exist poorly known, systematic,zonal effects which dilute the meaning of any "average". The table does, however, illustrate the unfortunate fact that the fundamental system deteriorates in the less observed southern sky. (Indeed the system could be legitimately described as a bi-modal system on an hemispherical basis). What is not shown is that the individual errors in the southern hemisphere fluctuate so widely that it is possible to encounter errors today which approach one second of arc.

If one omits the larger individual errors in the southern hemisphere, then it is correct to say that the FK4 system has an average error of a few hundredths of a second of arc at the mean epochs, and that this error increases at the rate of a few milli-arc seconds per year. 
What are the sources of these errors? There can be little doubt that systematic differences between contributing instruments is the prime source of error. The internal mean error of a single observation from a modern transit circle is \pm 0 ". 25 or less and is decreasing. A catalog Involving multiple observations of hundreds or thousands of stars, then, has formal errors perhaps between \pm 0 ":05 and \pm 0 "'10.

Without the presence of relatively large systematic errors one could combine several such catalogs and confidently expect an uncertainty measured in milli-arc seconds. This is patently not the case.

The source of systematic errors may be found both in the instruments and in their environments as well as in the cross coupling between the two. Transit circles are what might be called "micron-sensitive" instruments in the sense that micron sized displacements of lenses, pivots, circle graduations, micrometers, and so on, can and do produce important systematic effects as we11 as the more tractable random excursions. The effects of the environment are nowhere more evident than in seeing and refraction effects. One of the problems associated with many meridian observations (especially visual ones) is the lack of integration of information. A typical stellar position in the FK4 might be based upon (say) 500 single observations. The problem is that each of these observations might involve about 5 seconds of actual data recording. A simple calculation then shows that the entire fundamental system is based upon about 1,000 hours of actual data recording. This is equivalent to what, e.g. a radio astrometer might call several hundred single observations! Photoelectric trackers have addressed this problem with success ${ }^{5}$, and currently an integrating image dissector photon counting system is about to be tested by the author

Refraction not only directly influences stellar observations, but it also has pernicious indirect effects upon them via flexure, collimation and azimuth (mark) observations as well. As far as pure astronomical refraction is concerned, it is not likely that any new general theory (even one with many adjustable parameters) could do better than what is currently avallable. The atmosphere, and in particular the planetary boundary layer, is a dynamic medium whose intricacies are still being unravelled by meteorologists. Progress 
is being made in this area, however, by means of proposed multi-color measures on the one hand ${ }^{6}$, and by real time atmospheric probing on the other. In this manner site dependent environmental systematics ${ }^{7,8}$ may at last be minimized,if not eliminated.

A11 in all real progress is being made in classical meridian observing methods. Completely automated systems are being developed which incorporate improved circles and scanning methods (and envision inertial angle measurements), vastly improved focal plane measures, detailed specialized lens design and fabrication, plus other items including those mentioned previously

Even a brief review of the status of astrometry would be incomplete without mention of the photographic efforts. Comments here will be restricted to parallax and position determination.

A parallax can be "routinely" determined today with an error of about $\pm 0: 004$. If extended series of plates are used in conjunction with first class equipment and procedures, it is possible to reduce this to $\pm 0.002^{9}$. This latter figure is possible near the ultimate limit of the present methods.

A photographic star catalog is the result of a chaining process. Given a fundamental coordinate system as discussed earlier, it is necessary to observe fainter stars differentially with a transit circle in order to provide reference stars for the reduction of the astrographic plates. Using these fainter $\left(7<\mathrm{m}_{\mathrm{v}}<9\right)$ reference stars then, one can presumably extend the fundamental system out to the plate limit (say $\mathrm{m}_{\mathrm{v}} \sim 11$ ). In an absolute sense it is evident that a photographic catalog can never exceed the systematic accuracy of its reference stars. The formal mean errors of a good contemporary astrographic catalog are perhaps in the range \pm 0 ". 15 to \pm 0 ". 20 , but the interpretation of such errors is difficult. Very careful attention must be paid to the systematic errors depending upon magnitude and color. Modern optical design,coupled with increasingly accurate measuring engines, is helping to reduce the instrumental errors, while careful plate modeling and a more sophisticated treatment of the data (e.g. plate overlapping techniques) enable one to extract more of the information on the plate. 


\section{THE NEEDS OF ASTROMETRY}

The current fundamental system needs to be revised. The revision has already started ${ }^{10}$, and the new system, FK5, should be available in the early 1980 's. It would be presumptuous as this time to attempt to predict the quality of the FK5. However, since cosmologists and astrophysicists shouldn't have all the fun, the author speculates, (and it is just that) that the total mean error at epoch in the northern hemisphere will be decreased about $30 \%$ to $50 \%$, and that the decrease in the southern hemisphere, especially as regards the system error, will be a good deal less. This speculation is based upon the improvement achieved in replacing FK3 with FK4, and upon considerations of the new material available to the compilers of FK5.

An important fact is that FK5 will be expanded by the addition of perhaps as many as two thousand faint $\left(7<m_{v}<9\right)$ fundamental stars. This extension is very welcome, but it immediately presents us with another need....namely, that these fainter stars be continually observed. If they are not, the hemispherical bi-modality problem alluded to earlier will be extended into a magnitude bi-modality.

It has long been known that the precession constant given by Newcomb requires a correction. A new value has been derived ${ }^{11}$, and will become the standard for future observing programs. It is also likely that a new method for computing the nutation will be implemented at the same time. It will probably differentiate explicitly between "dynamical" and "geophysical" effects.

Not so very long ago the radio interferometer became an astrometric tool of great promise. Now, before the astrometrists (who are notoriously conservative) have really assimilated and assessed the possibilities and pitfalls of radio we are confronted by infra-red and optical interferometric techniques.

In general it can certainly be said that the ultimate need or, in reality, the raison d'etre, of fundamental astrometry is the production of an homogeneous coordinate system within which we can confidently apply Newton's Law (as modified by relativity if necessary). To accomplish this task it is absolutely necessary that we avoid fragmentation of our efforts. Coordinate 
systems are unavoidably dependent upon hardware, magnitude, wavelength, geometry, etc. and the whole history of astrometry involves attempts to break these correlations. If systems cannot be reliably transformed one to another their utility is marginal. The trick is to carefully consider the entire problem before one starts observing.

By way of a possible illustration, let us briefly consider the problem of using the superb accuracy of the radio interferometer to improve the fundamenta1 system. To this end, assume for the sake of argument that fifty to onehundred quasars have been observed, and that relative right ascensions and absolute declinations are available with an accuracy of \pm 0 '01 or even better. Since we are talking about 15 th to 18 th magnitude optical objects it is necessary to somehow relate this system to brighter objects. The astrograph is the logical choice, but from previous comments it is evident that the probability of conserving the \pm 0 '.01 down to even the twelfth magnitude (let alone the ninth) is very small. Thus, although we have reversed the classical information flow (from, bright $\rightarrow$ faint, to faint $\rightarrow$ bright) and markedly increased the accuracy of the standard in the bargain, the use of the resulting system must be carefully evaluated.

Astrometry finds itself today in the exciting position of an ex-wall flower besieged by handsome suitors from all sides. In addition to all the interferometric possibilities, one must consider also the use of dedicated astrometric satellites. The latter concept is emerging from the talking stage even now. All in all, there is no doubt that astrometry can only benefit greatly

\section{REFERENCES}

(1) G.M. Clemence, Proceedings of the Meeting on Problems of Astrometry and Celestial Mechanics, La Plata, 1961.

(2) R. Branham, private communication, 1978.

(3) W. Fricke and A. Kopff, "Fourth Fundamental Catalog (FK4),"

Veroff. Ast. Rechen-Institut, 1963.

(4) T. Lederle, Wiss. Z. Tech. Univ. Dresden, 25, 948, 1976. 
(5) E. Hфg, "New Problems in Astrometry" (I.A.U. Symposium No.61), W. Gliese, C.A. Murray, R.H. Tucker, eds., Reidel Publishing Co., Dordrecht, p.243, 1974.

(6) Currie, D., University of Maryland Techn. Rep. 77-076, 1977.

(7) J.A. Hughes, "New Problems in Astrometry" (I.A.U. Symposium No.61), W. Gliese, C.A. Murray, R.H. Tucker, eds., Reidel Publishing Co., Dordrecht, p.237, 1974.

(8) J.A. Hughes, "Refractional Influences in Astrometry and Geodesy" (I.A.U. Symposium No.89), in press.

(9) R. Harrington, private communication, 1978.

(10) W. Fricke, "New Problems in Astrometry" (I.A.U. Symposium No.61), W. Gliese, C.A. Murray, R.H. Tucker, eds., Reide1 Publishing Co., Dordrecht, p.1, 1974.

(11) J.H. Lieske, T. Lederle, W. Fricke and B. Morando, Astron. Astrophys., 58, 1,1977 .

\section{DISCUSSION}

P.L. Bender: I'd like to call attention to a proposal by Eichhorn and Williams that astrometric photography of a satellite which is also being tracked by ranging can give improvements in star positions. In addition, if the LAGEOS satellite is photographed with pulsed laser illumination while the Earth's angular position against the radio star reference frame (RRF) is monitored by VLBI, star positions can be determined in the RRF. The limitations are likely to be almost entirely those due to the astrometric photography and to the pulse to pulse seeing jitter for the laser-illuminated satellite.

J.A. Hughes: In addition to knowing where the origin is, the problem in photographic astrometry has always consisted of getting from point to point accurately. 